\title{
The Status of the Polarized Parton Densities
}

\author{
Johannes Blümlein * \\ Deutsches Elektronen-Synchrotron, DESY, Platanenallee 6, D-15738 Zeuthen, Germany
}

\begin{abstract}
A survey is given on the present knowledge of the polarized parton distribution functions. We give an outlook on further developments desired both on the theoretical as well on the experimental side to complete the understanding of the spin-structure of nucleons in the future.
\end{abstract}

\section{Introduction}

Deeply inelastic scattering provides a clean way to extract the parton densities of nucleons. After the initial observation that the nucleon spin is not formed by the quarks dominantly [2], detailed measurements of the polarized structure functions followed during the last 20 years. The central question concerns now the parton distribution functions and their scale evolution rather than just their first moment. Since the nucleon spin receives also contributions from the angular momentum of the quarks and gluons, these degrees of freedom have also to be studied. This requires the analysis of non-forward scattering cross sections. In inclusive deep-inelastic scattering the sensitivity to resolve the different sea-quark contributions is rather limited. One way to extract this information consists in measuring semi-inclusive processes [3]. A central question concerns the polarized gluon distribution, which can be determined from the scaling violations of $g_{1}\left(x, Q^{2}\right)$, deep-inelastic heavy flavor production, and hard processes measured at hadron colliders. The inclusive and semi-inclusive hard processes in polarized scattering provide an important laboratory to test QCD. More than exploring the level of twist-2, which has been investigated in some detail already, one may probe the twist -3 contributions in various transverse spin processes. In the following we survey the theoretical status of inclusive polarized deeply inelastic scattering and the status of polarized parton densities, cf. also [4]. We close with an outlook on investigations required in the future.

\section{Theoretical Aspects}

In the deep-inelastic domain the polarized nucleon structure functions receive contributions of leading and higher twist, depending on the region in $Q^{2}$ and $W^{2}$ probed. The leading twist contributions are those of twist $\tau=2$ for $g_{1}\left(x, Q^{2}\right)^{\text {a }}$ and $\tau=2,3$ for $g_{2}\left(x, Q^{2}\right)$. At the level of twist-2 one may extract the polarized parton densities from the data on the structure function $g_{1}\left(x, Q^{2}\right)$ performing an analysis in the framework of perturbative QCD. During the past decades higher orders have been approached steadily. The running of $\alpha_{s}\left(\mu^{2}\right)$ is known to $O\left(\alpha_{s}^{4}\right)$ [6], both the polarized anomalous dimensions [7] ${ }^{\mathrm{b}}$ and massless Wilson coefficients [9] were calculated to $O\left(\alpha_{s}^{2}\right)$ and the first non-singlet moment, the polarized Bjorken sum-rule, to $O\left(\alpha_{s}^{3}\right)$ [10]. The heavy flavor Wilson coefficients, in the whole kinematic region, are only known to $O\left(\alpha_{s}\right)$ [11]. For $Q^{2} \gg m^{2}$, i.e. in the region $Q^{2} / m^{2} \gtrsim 10$, the

\footnotetext{
*This paper was supported in part by SFB-TR-9: Computergestütze Theoretische Teilchenphysik.

a Note that $g_{1}\left(x, Q^{2}\right)$ contains twist $\tau=3$ contributions due to target mass corrections, [5].

${ }^{\mathrm{b}}$ Due to the Ward identity $P_{\mathrm{NS}}^{q q}=\Delta P_{\mathrm{NS}}^{q q}$ this splitting function is also known to $O\left(\alpha_{s}^{3}\right),[8]$.
} 
Wilson coefficients were calculated in $O\left(\alpha_{s}^{2}\right)$, [12]. An interesting property is exhibited by the gluonic heavy flavor Wilson coefficient, the first moment of which vanishes in leading and next-to-leading order $[11,12]$. Given a positive polarized gluon density, this implies a negative correction to $g_{1}\left(x, Q^{2}\right)$ in the region $x \lesssim 10^{-2}$ and a positive contribution above. Conversely, the Wandzura-Wilczek relation implies a positive correction for the twist-2 part of $g_{2}\left(x, Q^{2}\right)$ for $x \lesssim 2 \cdot 10^{-2}$, but a negative correction for larger $x$-values, cf. [13]. The anomalous dimensions for the evolution of the transversity distribution are known to $O\left(\alpha_{s}^{2}\right)$ in general [14] and for a series of moments to $O\left(\alpha_{s}^{3}\right)$ [15]. At present only next-to-leading order QCD analyzes can be performed to extract the polarized parton distributions.

At the level of the twist- 3 contributions to the polarized structure functions several sumrules and integral relations were derived, cf. $[5,16]$. The twist-2 contributions to the structure function $g_{2}\left(x, Q^{2}\right)$ is given by the Wandzura-Wilczek relation [17] for the quarkonic, gluonic, heavy flavor contributions, target mass corrections, and even diffractive scattering, cf. [5, 13, 18 . The twist -3 contributions to the structure function $g_{2}\left(x, Q^{2}\right)$ were calculated to oneloop order. The $O\left(\alpha_{s}\right)$ non-singlet and singlet anomalous dimension matrices, respectively their corresponding expressions in momentum fraction space, were derived in Refs. [19] using different techniques. The $O\left(\alpha_{s}\right)$ Wilson coefficients were calculated in [20]. Although the precision on $g_{2}\left(x, Q^{2}\right)$ improved during the last years [21] and some difference between the data and the Wandzura-Wilczek approximation is seen, still more precise data are required to test the QCD-predictions. First non-singlet moments of the twist-3 operator expectation values were determined in lattice simulations $[22,23]$.

Also in case of the polarized structure functions small- $x$ resummations are discussed, which can be described on the basis of infrared evolution equations [24] and emerge both for the non-singlet [25] and singlet structure functions [26,27]. These resummations apply to the leading pole $O\left(\left(\alpha_{s} / N^{3}\right)^{k}\right)$-terms only, with $N$ the Mellin-variable. Performing the resummation one obtains a branch-cut instead of the perturbative pole-terms, which yields a milder singularity. The resummation is consistently accounted for by the Callan-Symanzik equations for the evolution of parton densities. As detailed numerical studies, which were performed in Refs. [25,27], show ${ }^{\mathrm{c}}$, one has to take into account not only the leading pole terms, but also the resummed sub-leading terms, see also [29], which are not yet calculated completely. They are known, however, for the first two orders in $\alpha_{s}$ (and partly to $O\left(\alpha_{s}^{3}\right)$ ) for all sub-leading terms, which suggest the general form. The comparison of the leading and sub-leading terms shows, that at least three sub-leading terms are required to match the exact results. Ignorance of these contributions, as unfortunately still partly present in the contemporary literature, results into misleading quantitative analyzes. Sometimes also "DGLAP" evolution is opposed to "infrared evolution equations", etc. Here again a clarifying word is in order. In practice both concepts address twist-2 parton distributions. Their scale evolution results from the factorization of the collinear singularities and is ruled by the anomalous dimensions $P_{i j}\left(N, a_{s}\right)=\sum_{k=1}^{\infty} a_{s}^{k} P_{i j}^{(k-1)}(N)$. The corresponding CallanSymanzik equations have to be solved for high enough orders in the coupling constant in the range of Bjorken- $x$, demanded by the experimental data. These equations cover the small- $x$ and the less singular terms which are equally important in quantitative analyzes.

\footnotetext{
${ }^{\mathrm{c}}$ For the unpolarized case see [28].
} 


\section{Parton Distributions}

The polarized parton distribution functions may be determined by a QCD-analysis of the structure function $g_{1}\left(x, Q^{2}\right)$. The data analysis requires a detailed description of the denominator of the polarization asymmetry, which has to include empiric parameterizations both for $F_{2}\left(x, Q^{2}\right)$ and $F_{L}\left(x, Q^{2}\right)$ including potential higher twist contributions, since the region of $Q^{2}$ and $W^{2}$, which is analyzed, covers rather low values, unlike the case in unpolarized analyzes [30]. Usually one would like to limit the data analysis to the region $Q^{2} \gtrsim 4 \mathrm{GeV}^{2}$, which will be possible in future measurements at a facility like EIC [31]. The present data sets only allow a cut $Q^{2} \gtrsim 1 \mathrm{GeV}^{2}$. In the analysis the correlation of the different parameters of the parton distribution functions at $Q_{0}^{2}$ and the $\mathrm{QCD}$-scale $\Lambda_{\mathrm{QCD}}$ are rather essential. Measuring the gluon distribution function $\Delta G\left(x, Q^{2}\right)$, and to some extent also the sea-quark distributions, the slope effects of $\partial g_{1}\left(x, Q^{2}\right) / \partial \ln \left(Q^{2}\right)$ are important. In case of $\Delta G$ there one observes a very strong correlation with $\alpha_{s}\left(Q^{2}\right)$ due to the evolution equations. Special assumptions on $\Lambda_{\mathrm{QCD}}$, as fixing this value to other measurements, may introduce severe biases. In the inclusive analysis not all the parameters chosen to model the parton distributions can be measured. For the data sets currently available this applies in particular to those parameters which describe the range of medium values of $x$. Their error may be-
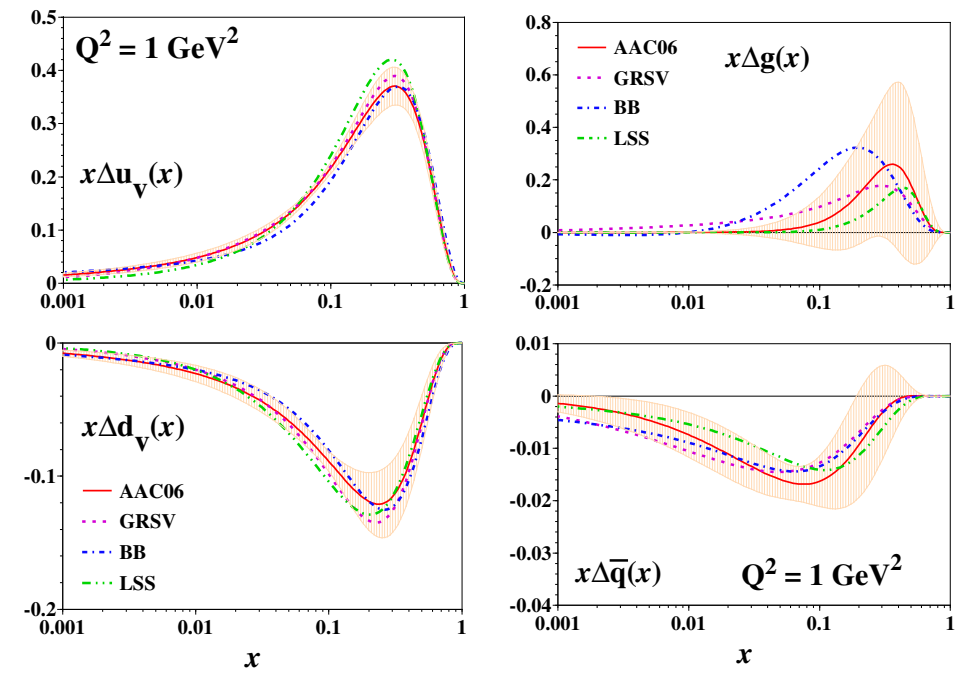

Figure 1: The polarized parton distributions from different analyzes at the scale $Q^{2}=1 \mathrm{GeV}^{2}$, [33]. AAC06 : [33]; GRSV : [34]; BB : [32]; LSS : [35].

come rather large compared to their value. These parameters can be fixed after a first minimization and form a certain model. Their value has to be re-fitted after the global minimum was found, but will usually not change significantly, cf. [32]. The relative normalization of the different data sets is fitted within the allowed margins quoted by the experiments, to account for global systematic effects. In Figure 1 recent parton distribution functions [32-35] are compared. Further parameterizations were given in [36-39]. Within the present errors there is good agreement between all analyzes. The valence quark distribution functions are determined best, with a positive polarized up-quark and and a negative down-quark distribution. The sea-quark distribution is found to be mainly negative, but with a larger error. To resolve the different flavors of the sea-quark contributions semi-inclusive data were analyzed [3], yet 
with rather large errors. Under certain assumptions the error on the strange-quark density becomes rather low [36]. Alternatively to the standard QCD fits neural-network techniques were used to determine the polarized parton densities in [38]. In this analysis a larger error than found using the conventional methods is obtained in the small $x$ region, were data are sparse. If compared to earlier analyzes [32] the polarized gluon distribution comes out at lower values including more recent data $[33,35,40]$. The polarized gluon distribution function, although being obtained with positive central values in unconstrained fits, is still compatible with zero within the errors. In some analyzes [35,39,41] one demands, as second option, also a negative normalization of the gluon distribution in a constrained fit. The corresponding distribution is slightly negative and allowed by the data under the constraint used. The ratio $\Delta G / G$ was also measured in open charm photo-production [42]. Here the experimental errors are still large and the result is compatible with zero. Using the fit results of the polarized parton distributions one may form moments, cf. [32], to be compared with lattice simulations, in particular for the valence-quark distributions. Here the crucial point is to control the systematic effects and to approach realistic values of the pion mass. Currently values in the range $m_{\pi} \sim 270 \mathrm{MeV}$ can be reached in dynamical quark simulations. In this way new non-perturbative quantitative test of QCD will be possible soon $[22,23,43]$.

\section{Future Avenues}

The current picture of the polarized nucleon is still in a move and more efforts in theory and experiment are needed to complete it. In the forthcoming years the data-analysis from HERMES and COMPASS will lead to still better parton distribution functions. It would be important to measure the structure function $F_{2}\left(x, Q^{2}\right)$ at HERMES, which would yield an improved systematic understanding of the data. Yet the experimental precision of the structure function $g_{1}\left(x, Q^{2}\right)$ is limited and high-luminosity measurements at a future facility as EIC [31] is highly desirable to provide detailed QCD-tests for $\Lambda_{\mathrm{QCD}}$, the parton distributions and their moments to be compared with lattice simulations. The experiments at RHIC will improve our knowledge on the polarized sea-quark and gluon distributions. Important information on the large- $x$ behaviour of all distribution functions, can be gained in the experiments at JLAB running at an increased beam energy. The HERA experiments, COMPASS and the JLAB experiments will finalize their measurements on deeply-virtual Compton scattering (DVCS) [44] during the forthcoming years and we may hope to get constraints on the quark angular momentum [45] using Ji's sum-rule [46].

As shown in [47] the theory error of the polarized gluon distribution at NLO is still large. The calculation of the 3-loop anomalous dimensions are therefore required to diminish this uncertainty. Very high statistics measurements have to be performed in the long-term future for DVSC to extract constraints on the angular momentum of the gluon from the scaling violations of the non-forward scattering cross sections. As is well-known, the scaling violations of the transversity distribution function $h_{1}\left(x, Q^{2}\right)$ are larger than those of the nonsinglet part of $g_{1}\left(x, Q^{2}\right)$. Detailed high statistics measurements of this quantity are desirable to establish this prediction of QCD experimentally. Very little is known about the higher twist contributions to polarized deeply-inelastic scattering. Here we may hope for results from the experiments at JLAB. For the general kinematic region again high-luminosity experiments as planned for EIC would provide an excellent opportunity. Dedicated studies of the twist- 3 contributions to several processes should be carried out and measurements 
shall be performed to isolate the twist- 4 contributions. An interesting open issue is formed by twist-3 effects $[5,16]$ in deep-inelastic scattering in electro-weak interactions, which can be studied at future neutrino factories [48]. The present status of our knowledge on polarized parton densities is not yet sufficient and calling for refined measurements in various places which are crucial for the final understanding of the spin-structure of the nucleons. This will require extensive experimentation at a high-luminosity facility such as the future ElectronIon-Collider.

\section{References}

[1] Slides: http: //indico. cern. ch/contributionDisplay py? contribId=71\&sessionId=15\&conf Id=9499

[2] M. J. Alguard et al. (SLAC), Phys. Rev. Lett. 37 (1976) 1261; 41 (1978) 70.

[3] A. Airapetian et al. [HERMES Collaboration], Phys. Rev. D 71 (2005) 012003.

[4] J. Blümlein, arXiv:hep-ph/0510212.

[5] J. Blümlein and A. Tkabladze, Nucl. Phys. B 553 (1999) 427.

[6] T. van Ritbergen, J. A. M. Vermaseren and S. A. Larin, Phys. Lett. B 400 (1997) 379; M. Czakon, Nucl. Phys. B 710 (2005) 485.

[7] R. Mertig and W. L. van Neerven, Z. Phys. C 70 (1996) 637; W. Vogelsang, Nucl. Phys. B 475 (1996) 47; Phys. Rev. D 54 (1996) 2023.

[8] S. Moch, J. A. M. Vermaseren and A. Vogt, Nucl. Phys. B 688 (2004) 101.

[9] E. B. Zijlstra and W. L. van Neerven, Nucl. Phys. B 417 (1994) 61 [Erratum-ibid. B 426 (1994) 245; 773 (2007) 105].

[10] S. A. Larin and J. A. M. Vermaseren, Phys. Lett. B 259 (1991) 345.

[11] A. D. Watson, Z. Phys. C 12 (1982) 123.

[12] M. Buza, Y. Matiounine, J. Smith and W. L. van Neerven, Nucl. Phys. B 485 (1997) 420; J. Blümlein and S. Klein, DESY 07-027;

I. Bierenbaum, J. Blümlein and S. Klein, arXiv:0706.2738 [hep-ph].

[13] J. Blümlein, V. Ravindran and W. L. van Neerven, Phys. Rev. D 68 (2003) 114004.

[14] S. Kumano and M. Miyama, Phys. Rev. D 56 (1997) 2504;

A. Hayashigaki, Y. Kanazawa and Y. Koike, Phys. Rev. D 56 (1997) 7350; W. Vogelsang, Phys. Rev. D 57 (1998) 1886.

[15] J. A. Gracey, Phys. Lett. B 643 (2006) 374; JHEP 0610 (2006) 040.

[16] J. Blümlein and N. Kochelev, Nucl. Phys. B 498 (1997) 285;

[17] S. Wandzura and F. Wilczek, Phys. Lett. B 72 (1977) 195.

[18] J. D. Jackson, G. G. Ross and R. G. Roberts, Phys. Lett. B 226 (1989) 159;

J. Blümlein and N. Kochelev, Phys. Lett. B 381 (1996) 296;

A. Piccione and G. Ridolfi, Nucl. Phys. B 513 (1998) 301;

J. Blümlein and D. Robaschik, Phys. Rev. D 65 (2002) 096002;

J. Blümlein, B. Geyer and D. Robaschik, Nucl. Phys. B 755 (2006) 112; arXiv:0706.2478 [hep-ph].

[19] E. V. Shuryak and A. I. Vainshtein, Nucl. Phys. B 201 (1982) 141;

A. P. Bukhvostov, E. A. Kuraev and L. N. Lipatov, JETP Lett. 37 (1983) 482 [Pisma Zh. Eksp. Teor. Fiz. 37 (1983 SPHJA,60,22-32.1984 ZETFA,87,37-55.1984) 406];

A. P. Bukhvostov, G. V. Frolov, L. N. Lipatov and E. A. Kuraev, Nucl. Phys. B 258 (1985) 601;

P. G. Ratcliffe, Nucl. Phys. B 264 (1986) 493;

I. I. Balitsky and V. M. Braun, Nucl. Phys. B 311 (1989) 541;

X. D. Ji and C. h. Chou, Phys. Rev. D 42 (1990) 3637;

A. Ali, V. M. Braun and G. Hiller, Phys. Lett. B 266 (1991) 117;

J. Kodaira, Y. Yasui and T. Uematsu, Phys. Lett. B 344 (1995) 348;

J. Kodaira, Y. Yasui, K. Tanaka and T. Uematsu, Phys. Lett. B 387 (1996) 855;

J. Kodaira, T. Nasuno, H. Tochimura, K. Tanaka and Y. Yasui, Prog. Theor. Phys. 99 (1998) 315;

B. Geyer, D. Müller and D. Robaschik, Nucl. Phys. Proc. Suppl. 51C (1996) 106;

D. Müller, Phys. Lett. B 407 (1997) 314;

V. M. Braun, G. P. Korchemsky and A. N. Manashov, Phys. Lett. B 476 (2000) 455; Nucl. Phys. B 597 (2001) 370; B 603 (2001) 69. 
[20] X. D. Ji, W. Lu, J. Osborne and X. T. Song, Phys. Rev. D 62 (2000) 094016; A. V. Belitsky, X. D. Ji, W. Lu and J. Osborne, Phys. Rev. D 63 (2001) 094012.

[21] X. Zheng et al. [Jefferson Lab Hall A Collaboration], Phys. Rev. C 70 (2004) 065207.

[22] M. Göckeler et al., [QCDSF collaboration], Phys. Rev. D 72 (2005) 054507; H. W. Lin, arXiv:0707.3844 [hep-lat].

[23] D. Dolgov et al. [LHPC collaboration], Phys. Rev. D 66 (2002) 034506.

[24] R. Kirschner and L. N. Lipatov, Nucl. Phys. B 213 (1983) 122.

[25] J. Blümlein and A. Vogt, Phys. Lett. B 370 (1996) 149;

J. Blümlein, S. Riemersma and A. Vogt, Nucl. Phys. Proc. Suppl. 51C (1996) 30.

[26] J. Bartels, B. I. Ermolaev and M. G. Ryskin, Z. Phys. C 72 (1996) 627.

[27] J. Blümlein and A. Vogt, Phys. Lett. B 386 (1996) 350.

[28] J. Blümlein, V. Ravindran, W. L. van Neerven and A. Vogt, arXiv:hep-ph/9806368; J. Blümlein and A. Vogt, Phys. Rev. D 58 (1998) 014020; D 57 (1998) 1.

[29] J. Blümlein and W. L. van Neerven, Phys. Lett. B 450 (1999) 412.

[30] A. D. Martin, R. G. Roberts, W. J. Stirling and R. S. Thorne, Eur. Phys. J. C 35 (2004) 325; J. Pumplin, A. Belyaev, J. Huston, D. Stump and W. K. Tung, JHEP 0602 (2006) 032;

S. Alekhin, K. Melnikov and F. Petriello, Phys. Rev. D 74 (2006) 054033;

J. Blümlein, H. Böttcher and A. Guffanti, Nucl. Phys. B 774 (2007) 182;

J. Blümlein, arXiv:0706.2430 [hep-ph].

[31] C. Aidala et al. [EIC Working Group], A White Paper Prepared for the NSAC LPR 2007, A High Luminosity, High Enery Electron-lon-Collider.

[32] J. Blümlein and H. Böttcher, Nucl. Phys. B 636 (2002) 225.

[33] M. Hirai, S. Kumano and N. Saito, Phys. Rev. D 74 (2006) 014015.

[34] M. Glück, E. Reya, M. Stratmann and W. Vogelsang, Phys. Rev. D 63 (2001) 094005.

[35] E. Leader, A. V. Sidorov and D. B. Stamenov, Phys. Rev. D 73 (2006) 034023.

[36] D. de Florian, G. A. Navarro and R. Sassot, Phys. Rev. D 71 (2005) 094018.

[37] S. Atashbar Tehrani and A. N. Khorramian, JHEP 0707 (2007) 048.

[38] A. Guffanti, talk at SPIN06, Kyoto, 2006;

J. Rojo et al. [NNPDF Collaboration], arXiv:0706.2130 [hep-ph];

L. Del Debbio, S. Forte, J. I. Latorre, A. Piccione and J. Rojo [NNPDF Collaboration], JHEP 0703 (2007) 039.

[39] V. Y. Alexakhin et al. [COMPASS Collaboration], Phys. Lett. B 647 (2007) 8.

[40] J. Blümlein and H. Böttcher, in preparation.

[41] K. Kurek, these proceedings.

[42] K. Kurek, arXiv:hep-ex/0607061;

G. K. Mallot, arXiv:hep-ex/0612055;

S. Koblitz [COMPASS Collaboration], arXiv:0707.0175 [hep-ex].

[43] Ph. Hägler et al. [LHPC Collaborations], arXiv:0705.4295 [hep-lat];

W. Schroers, Eur. Phys. J. A 31 (2007) 784;

R. G. Edwards et al. [LHPC Collaboration], Phys. Rev. Lett. 96 (2006) 052001;

A. A. Khan et al., Phys. Rev. D 74 (2006) 094508;

K. Jansen et al., in preparation.

[44] A. V. Belitsky and A. V. Radyushkin, Phys. Rept. 418 (2005) 1 and references therein.

[45] F. Ellinghaus, W. D. Nowak, A. V. Vinnikov and Z. Ye, Eur. Phys. J. C 46 (2006) 729.

[46] X. D. Ji, Phys. Rev. Lett. 78 (1997) 610.

[47] J. Blümlein and H. Böttcher, Nucl. Phys. A 721 (2003) 333.

[48] M. L. Mangano et al., arXiv:hep-ph/0105155. 